\title{
Hybrid Genetic Algorithm and Particle Swarm Optimization Algorithm for Optimal Power Flow in Power System
}

\author{
Gayathri Devi K.S \\ Noorul Islam Center for Higher Education, \\ Kumaracoil, Tamil Nadu, India \\ ksgayathridevi2@gmail.com
}

\begin{abstract}
In power systems, the Optimal Power Flow (OPF) problem is considered as the majority extensively nonlinear optimization problems. This work presents a new hybrid optimization approach that integrates the advantages of the Genetic Algorithm (GA) with the Particle Swarm Optimization (PSO) approach to solve the OPF problem. The developed hybrid algorithm is considered to achieve environmental, technical, and economic, advantages. The proposed technique is used to single and multi-objective optimization problems using diverse objective models like minimization of generation cost, reduction of emission, minimization of transmission power loss, maximization of voltage profile, and maximization of voltage stability. To show the ability of developed hybrid optimization method, single-objective cases are used and verified on three standard bus systems. The developed PSO-GA technique attains considerably the efficiency, and the robustness of OPF outcomes for the cases, are represented. The experimentation outcomes show that the developed technique tends to better levels of techno-economic-environmental advantages evaluated. Additionally, the sensitivity analysis study verifies that the proposed hybrid algorithm constructs robust outcomes over parameter variations.
\end{abstract}

Keywords: Power System; OPF; Emission; Voltage Profile; Optimization Algorithm

\begin{tabular}{ll} 
Nomenclature \\
\hline Abbreviations & Descriptions \\
\hline OPF & Optimal power flow \\
JADE & Enhanced Adaptive Differential Evolution \\
SCOPF & Security Constrained Optimal Power Flow \\
CR & Crossover Rate \\
AMTPG-Jaya & Adaptive Multiple Teams Perturbation-Guiding Jaya \\
PDIPM & Primal-Dual Interior Point Method \\
HBE & Heat Balance Equations \\
OPFCTTB & OPF Considering Transient Thermal Behavior \\
OPF & Optimal power flow \\
JADE & Enhanced Adaptive Differential Evolution \\
SCOPF & Security Constrained Optimal Power Flow \\
\hline
\end{tabular}

\section{Introduction}

$\mathrm{OPF}$ is an essential statistical optimization method to make decisions in power system planning and operation. Enhancing the OPF solution quality can provide huge economic and engineering advantages to the power industry. Current research concentrating on the convexification of the OPF model undergoes from imprecision because of the recreation of numerous restraints [1].

Electric power companies are continually looking for cost-efficient operational situations by optimizing additional objectives as preserving varied systems restraints. Now, OPF solution is an important stratagem for contemporary power grids control and operation. It is a tremendously high-level, non-linear, multi-dimensional, and limited optimization problem. The explanation task of OPF solution is to regularize steady circumstance operation parameters of complete electrical elements obtainable in an energy system whereas very important objectives are supposed to optimize. 
The main purpose of the OPF problem is to attain optimal settings of control variables that optimize the chosen objective model whilst fulfilling different power flow formulations and inequality restraints in a specified power system network. The generally exploited objective function is the reduction cost function of fuel, active power loss, emission, and deviation of bus voltage. Whereas the control variables of a power system, such as generators bus voltage magnitudes, real power outputs, and shunt capacitor outputs and so on, are operated to attain an optimal network setting [3] [4]. Because of the detail in which there are numerous control variables, and not all, are incessant, such as the shunt capacitor outputs, OPF problem is believed as a multi-modal, non-convex, large-scale, and non-linear constrained optimization problem [13] [14] [15]. In the interim, this as well creates resolving the OPF problem a significant problem in power system optimization [2].

The traditional formulation of OPF optimizes nodal complex voltages athwart a presented network regarding definite principle (for example., cost of generation) cause to experience quadratic equations based on Kirchhoff's law, power conservation, line thermal limits, and voltage stability limitations, amid other constraints. Because of the intrinsic physical laws complexity which method electricity flow, a few of these constraints are non-convex that produces OPF problem NP-hard generally. One alternative of this vital problem is security-constrained OPF whereas one encompasses based on an emergency on network modules too. Other than cost minimization, security-constrained OPF along with plans for alternative schemes with the intention that based on the failure, in-service generators can recompense by altered their outputs. In a few other alternatives to this problem such as security-constrained unit commitment, unexploited generators are integrated with altering in demand and to alleviate contingencies. Various other additions of OPF was examined in more wide-ranging settings, to tackle considerations like the operation security, energy storage, robustness, distributed platforms, generation uncertainty hybrid AC-DC grids, data-driven OPF, voltage stability, real-time operation, discrete aspects like transformer tap locations and unit commitment, between others.

The most important contribution of the paper is to propose a hybrid optimization technique will be performed by integrating the advantages of two optimization methods such as GA and PSO, to solve the OPF problem. This proposed method is used and examined on three bus test systems such as IEEE 30, 57, and 118-bus systems. To show the efficiency of the developed technique, experimentation outcomes are evaluated and critically examined over constraint violations such as individual development of GA and PSO. The hybrid algorithm indicates the continuous and discrete control variables.

\section{Literature Review}

In 2020, Zhao Yuan and Mario Paolone [1], developed a branch capacity constraint else transmission line derivation for the losses of power. It proves that the SOC-ACOPF technique with the extra constraint to develop its possibility was a comfortable ACOPF model. A possible solution revival process was derived while the SOC recreation was taut. The parametric best value functions of SOC-ACOPF and o-ACOPF techniques were shown monotonic about concerning power loads while the objective model was monotonic about concerning power generations. Larger power loads can make tighter the recreation in second-order cone ACOPF SOC-ACOPF model was proven.

In 2020, Shuijia Li et al [2], proposed a JADE about the self-adaptive penalty constraint handling method, indicated as EJADE-SP, to attain the best solution of OPF problem. The proposed method was an improved adaptation of JADE, whereas four enhancements were developed to improve the JADE performance while resolving the OPF problem: CR sorting model was developed to permit individuals to inherit additional superior genes; re-randomizing parameters (CR and scale factor) to preserve the search competence and variety; dynamic population lessening approach was exploited to accelerate convergence, and self-adaptive penalty constraint handling method was combined to tackle about constraints.

In 2020, Hossein Saberi et al [3], developed the SCOPF problem withDC load flow formulations was extensive to regard as the transient stability margin of generating units as a heuristic decomposition method. Nevertheless, the developed technique was companionable with any SCOPF equation. Because of the computational complexity of security constraints, the Benders decomposition method and transient stability evaluation were used to tackle the transient stability margin of generating units as separate sub-problems.

In 2019, Fariba Zohrizadeh et al [4], reviews the most recent research to show the achievement of conic optimization while used to power systems. The most important focal point was on how linear programming, second-order cone programming, and semi definite programming were exploited to addresses a central problem called the OPF problem. At last, interior-point techniques, first-order techniques, and nonconvex techniques were presented to solve conic optimization. 
In 2020, Warid Warid [5], developed the accomplishment of a lately adopted meta-heuristic optimization resolver called, an AMTPG-Jaya method to deal with varied single objective OPF models. The AMTPG-Jaya resolver uses many populations called teams to examine the search domain. The technique alters the number of teams beside with the forthcoming to supreme thus far nominee solution. Here, a conventional AMTPG-Jaya enthused algorithm to tackle with the OPF formulation was recommended.

In 2020, Mengxia Wang et al [6], developed the OPFCTTB model of transparency lines and the solution techniques. The OPFCTTB model was recognized by developed the HBEs of overhead lines as equality constraints and line temperature to make a variable decision into the AC OPF technique. To resolve the OPFCTTB technique which constraints comprise of nonlinear differential-algebraic formulations, the technique was initially discretized interested in a dynamic optimization problem whose restraints were algebraic formulations, and subsequently, the PDIPM was used to resolve the discretized technique.

\section{Objective Model}

In the power system, the OPF is a non-convex, non-linear, optimization problem that reduces definite objectives dependent on operational inequality and equality constraints. To solve OPF indicates a wellliked however demanding mission between power system studies. The very important task of OPF is to optimize the performance of the power system whereas fulfilling inequality and equality constraints.

\subsection{Objective functions}

The wide-ranging mathematical expression of the OPF problem deliberating $n$ - objective function can be indicated as [7]:

Dependent on:

$$
\min f_{y}=\left\{f_{1}(y), f_{2}(y), f_{3}(y), \ldots . . . f_{n}(y)\right\}
$$

$$
\begin{aligned}
& \mathrm{G}(\mathrm{y}) \geq 0, \mathrm{i}=0,1, \ldots \mathrm{m} \\
& \mathrm{H}_{\mathrm{i}}(\mathrm{y}) \geq 0, \mathrm{i}=0,1, \ldots . \mathrm{p} \\
& \mathrm{L}_{\mathrm{i}} \leq \mathrm{y}_{\mathrm{i}} 0 \leq \mathrm{U}_{\mathrm{i}}, \mathrm{i}=0,1, \ldots . \mathrm{n}
\end{aligned}
$$

Eq. (1) indicates the objective functions whilst eq. 2-4 presents operational inequality, equality, and boundary constraints. The OPF objective models can categorize as economic, technical, and environmental objectives. The cost of fuel minimization is considered the most important economical objective model. On the technical side, 3 objectives are represented; minimization of general transmission active power losses; developing voltage profile, and developing voltage stability. At last, the minimization of total emission is indicated as an environmental objective. The mathematical formulations of predefined objectives are explained as below:

The most significant objective model of the OPF problem is the minimization of total fuel costs of the generated real power of interlinked units. The quadratic function indication of costs of fuel $F_{1}$ is represented as below:

$$
\min \mathrm{F}_{1}=\sum_{\mathrm{i}=1}^{\mathrm{N}_{\mathrm{g}}} \mathrm{a}_{\mathrm{i}} \mathrm{P}_{\mathrm{g}_{\mathrm{i}}}^{2}+\mathrm{bP}_{\mathrm{g}_{\mathrm{i}}}+\mathrm{c}_{\mathrm{i}} \$ / \mathrm{hr}
$$

The second significant objective model of the OPF problem is the minimization of transmission real power loss $\mathrm{F}_{2}$ that is indicated in eq. (6).

$$
\min \mathrm{F}_{2}(\mathrm{y})=\sum_{\mathrm{i}=1}^{\mathrm{N}}\left(\mathrm{G}_{\mathrm{k}}\left(\mathrm{U}_{\mathrm{i}}^{2}+\mathrm{U}_{\mathrm{j}}^{2}-2 \mathrm{U}_{\mathrm{i}} \mathrm{U}_{\mathrm{j}} \cos \delta_{\mathrm{ij}}\right)\right)
$$

The 3rd objective is the minimization of voltage deviation at load buses is stated in eq. (7).

$$
\mathrm{F}_{3}(\mathrm{y})=\Delta \mathrm{U}=\sum_{\mathrm{i}=1}^{\text {Nbus }}\left|\mathrm{U}_{\mathrm{i}}-1\right|
$$

The emission objective is stated in eq. (8).

$$
\mathrm{F}_{4}=\sum_{\mathrm{i}=1}^{\mathrm{NG}} 10^{-2}\left(\alpha_{\mathrm{i}}+\beta_{\mathrm{i}} \mathrm{P}_{\mathrm{gi}_{\mathrm{i}}}+\gamma \mathrm{P}_{\mathrm{gi}}^{2}\right)+\mid \xi_{\mathrm{i}} \exp \left[\lambda_{\mathrm{i}} \mathrm{P}_{\mathrm{gi}_{\mathrm{i}}}\right]
$$

To improve the voltage stability system, it has aspired for reduction of Voltage Stability Index (Lindex), it is stated as follows [8]: 
Hybrid Genetic Algorithm and PSO Algorithm for OPF in Power System

$$
\begin{aligned}
\mathrm{L}_{\mathrm{i}} & =\left|1-\sum_{\mathrm{i}=1}^{\mathrm{N}_{\mathrm{g}}} \mathrm{F}_{\mathrm{ji}} \frac{\mathrm{U}_{\mathrm{i}}}{\mathrm{U}_{\mathrm{j}}}<\left(\theta_{\mathrm{ij}}+\delta_{\mathrm{i}}-\delta_{\mathrm{j}}\right)\right| \\
\mathrm{F}_{\mathrm{ji}} & =-\left[\mathrm{X}_{\mathrm{LL}}\right]^{-1}\left[\mathrm{X}_{\mathrm{LG}}\right]
\end{aligned}
$$

Hence, the 5th objective function aspires for minimization of utmost L-index in the system is represented in eq. (11).

$$
F_{5}=\operatorname{Max}\left(L_{j}\right) \quad j=1,2, \ldots \ldots, N_{b}
$$

\subsection{Operational constraints}

The preceding objectives are given to operational inequality and equality constraints. The reactive and active power flow power balance formulations are represented as below:

$$
\begin{gathered}
\mathrm{Q}_{\mathrm{g}_{\mathrm{i}}}-\mathrm{Q}_{\mathrm{L}_{\mathrm{i}}}+\mathrm{Q}_{\mathrm{C}_{\mathrm{i}}}-\mathrm{U}_{\mathrm{i}} \sum_{\mathrm{j}=1}^{\mathrm{N}_{\mathrm{b}}} \mathrm{U}_{\mathrm{j}}\left(\mathrm{G}_{\mathrm{ij}} \sin \theta_{\mathrm{ij}}-\mathrm{B}_{\mathrm{ij}} \cos \theta_{\mathrm{ij}}\right)=0, \mathrm{i}=1,2, \ldots \mathrm{N}_{\mathrm{PQ}} \\
\mathrm{P}_{\mathrm{gi}_{\mathrm{i}}}-\mathrm{P}_{\mathrm{L}_{\mathrm{i}}}+\mathrm{Q}_{\mathrm{c}_{\mathrm{i}}}-\mathrm{U}_{\mathrm{i}} \sum_{\mathrm{j}=1}^{\mathrm{N}_{\mathrm{b}}} \mathrm{U}_{\mathrm{j}}\left(\mathrm{G}_{\mathrm{ij}} \sin \theta_{\mathrm{ij}}+\mathrm{B}_{\mathrm{ij}} \cos \theta_{\mathrm{ij}}\right)=0, \mathrm{i}=1,2, \ldots . \mathrm{N}_{\mathrm{b}}-\text { slack }
\end{gathered}
$$

The inequality constraints are represented as below:

(a) Generators constraints:

$$
\begin{aligned}
& \mathrm{P}_{\mathrm{g}_{\mathrm{i}}}^{\min } \leq \mathrm{P}_{\mathrm{g}_{\mathrm{i}}} \leq \mathrm{P}_{\mathrm{gi}_{\mathrm{i}}}^{\max } \\
& \mathrm{Q}_{\mathrm{g}_{\mathrm{i}}}^{\min } \leq \mathrm{Q}_{\mathrm{g}_{\mathrm{i}}} \leq \mathrm{Q}_{\mathrm{g}_{\mathrm{i}}}^{\max } \\
& \mathrm{U}_{\mathrm{g}_{\mathrm{i}}}^{\min } \leq \mathrm{U}_{\mathrm{g}_{\mathrm{i}}} \leq \mathrm{U}_{\mathrm{gi}_{\mathrm{i}}}^{\max }
\end{aligned}
$$

(b) Transformer constraints (tapping):

$$
\mathrm{T}_{\mathrm{I}}^{\min } \leq \mathrm{T}_{\mathrm{i}} \leq \mathrm{T}_{\mathrm{i}}^{\max }
$$

(c)Voltage magnitude constraints of load buses:

$$
\mathrm{U}_{\mathrm{L}_{\mathrm{I}}}^{\min } \leq \mathrm{U}_{\mathrm{Li}} \leq \mathrm{U}_{\mathrm{Li}}^{\max }
$$

(d) Power flow constraints of transmission lines:

$$
\mathrm{S}_{\mathrm{L}_{I}}^{\min } \leq \mathrm{S}_{\mathrm{Li}} \leq \mathrm{S}_{\mathrm{Li}}^{\max }
$$

\section{Hybrid Genetic and PSO Algorithm for Optimal Power Flow}

\subsection{Conventional-Genetic Algorithm}

GA is a computational technique employed to emulate the presumption of biological evolution developed in [9]. It is an effectual global parallel optimization computation approach [10]. GA exploits the population search model and proposes a series of genetic operations on the present population, like selection, crossover, and mutation to produce a novel generation of population, and slowly develops the population to a condition which comprises or techniques the optimal solution.

Initially, initialize the population and computes the fitness model chosen by the individual, subsequently filer individuals during selection, mutation, and a crossover, with the intention that individuals with better fitness values are preserved, individuals about pitiable fitness are eradicated. The novel group not only innate previous generation information, other than outperforms preceding generation.

Here, the roulette technique and the real number cross technique is used in selection and cross operations. The selection probability of every individual is:

$$
p_{i}=\frac{f_{i}}{\sum_{i=1}^{N} f_{i}} ; f_{i}=\frac{k}{F_{i}}
$$

In eq. (20), $\mathrm{k}$ is the coefficient and $\mathrm{F}_{\mathrm{i}}$ indicates the fitness value. The chromosome crossing technique is stated in eq. (21). 
Hybrid Genetic Algorithm and PSO Algorithm for OPF in Power System

$$
\left\{\begin{array}{l}
b_{k j}=b_{k j} \cdot(1-a)+b_{i j} \cdot a \\
b_{1 j}=b_{1 j} \cdot(1-a)+b_{k j} \cdot a
\end{array}\right.
$$

At last, $\mathrm{j}^{\text {th }}$ gene of $\mathrm{i}^{\text {th }}$ individual is selected to mutate:

$$
b_{i j}= \begin{cases}b_{i j}+\left(b_{i j}-b_{\text {max }}\right) \cdot f(g) & r>0.5 \\ b_{1 j}+\left(b_{\text {min }}-b_{i j}\right) \cdot f(g) & r \leq 0.5\end{cases}
$$

In eq. (22), g indicates the current iteration number, $\mathrm{r}$ indicates a random number, $\mathrm{G}_{\max }$ indicates the highest number of evolutions

$$
\mathrm{f}(\mathrm{g})=\mathrm{r}\left(1-\mathrm{g} / \mathrm{G}_{\max }\right)^{2}
$$

\subsection{Conventional-PSO Approach}

The PSO technique is a bionic approach developed in [11] that is enthused by the behavior of the bird population. In general, at the time of the collective predation procedure, diverse birds can share huge information with every other. By this information sharing technique, birds will arrive at a few implicit thoughtful and lastly discover whereas the food is presented.

A particle is a possible solution for the optimization problem to be resolved. Throughout the flight, particles can exploit their own flight knowledge and the group's flight occurrence to dynamically regulate them. After numerous iterative searches, the best solution can be attained.

The PSO is initialized as a flock of arbitrary particles, and subsequently, the optimal solution is established during iteration. Particles update themselves by following the great value of individuals and flocks. While every particle discovers the best values, it updates its individual speed and a novel position by Eq. (24) and (25) in each iteration.

$$
\begin{aligned}
& \mathrm{U}_{\mathrm{k}+1}=\mathrm{u}_{\mathrm{k}}+\mathrm{c}_{1}\left(\mathrm{P}_{\text {best }_{\mathrm{k}}}-\mathrm{y}_{\mathrm{k}}\right)+\mathrm{c}_{2}\left(\mathrm{G}_{\text {best }_{\mathrm{k}}}-\mathrm{y}_{\mathrm{k}}\right) \\
& \mathrm{y}_{\mathrm{k}+1}=\mathrm{y}_{\mathrm{k}}+\mathrm{u}_{\mathrm{k}+1}
\end{aligned}
$$

whereas, $u_{k}$ indicates the particle velocity vector, $P_{b} t_{k}$ indicates the location of the best solution found by particle itself, $\mathrm{y}_{\mathrm{k}}$ indicates the current particle location, and $\mathrm{G}_{\mathrm{best}_{\mathrm{k}}}$ indicates optimal solution position established by the groups; $c_{2}$ and $c_{1}$ indicates "social-cognition factor" and "self-cognition factor" of particles, they are exploited to regulate the intensity of influence of $\mathrm{P}_{\text {best }_{\mathrm{k}}}$ and $\mathrm{G}_{\text {best }_{\mathrm{k}}}$ on the attraction of the particles, and the values are both arbitrary information between $[0,2]$.

\subsection{Hybrid GA-PSO Approach}

Here, GA and PSO are used and appropriate it to parameter fitting and fig 1 shows the flowchart of the Hybrid proposed model. The genetic operator is entrenched in the PSO method to progress the balance among exploration and expansion abilities.

Initially, the PSO population is initialized, and subsequently, the individual particle swarm fitness value is computed. The individual best particle and group optimal particle is updated based on particle fitness value. After producing a novel generation in PSO iteration, the present population is restored with an improved population via Genetic Algorithm operations like selection, mutation, and a crossover, and after that, a novel population is produced [12].

\section{Results and Discussions}

\subsection{Experimental Procedure}

In this paper, to confirm the efficacy of the developed algorithm, three test power systems were deliberated. These systems are; IEEE 30-, 57- and 118-bus test systems. Here, the developed method was evaluated with conventional algorithms such as PSO and GA algorithms.

\subsection{Performance Analysis}

Table 1, 2, and 3 show the results of the objective functions for the proposed and conventional methods for IEEE 30, 57, and 118-bus test systems. Here, the fuel costs, voltage deviation level, power losses, Lmax, and emissions are considered as the objective model. The developed technique presents the minimum cost, minimum voltage deviation level. It causes an improved voltage profile with enhanced evaluated with the conventional PSO and GA methods. The proposed method possesses minimized power 
losses over conventional methods. The stability index Lmax is reduced to enhance system stability. The emissions are considered as the final objective function to convene the environmental needs. It is obvious that the developed method tends to the highest power losses minimization and most improved voltage profile and economical solutions.

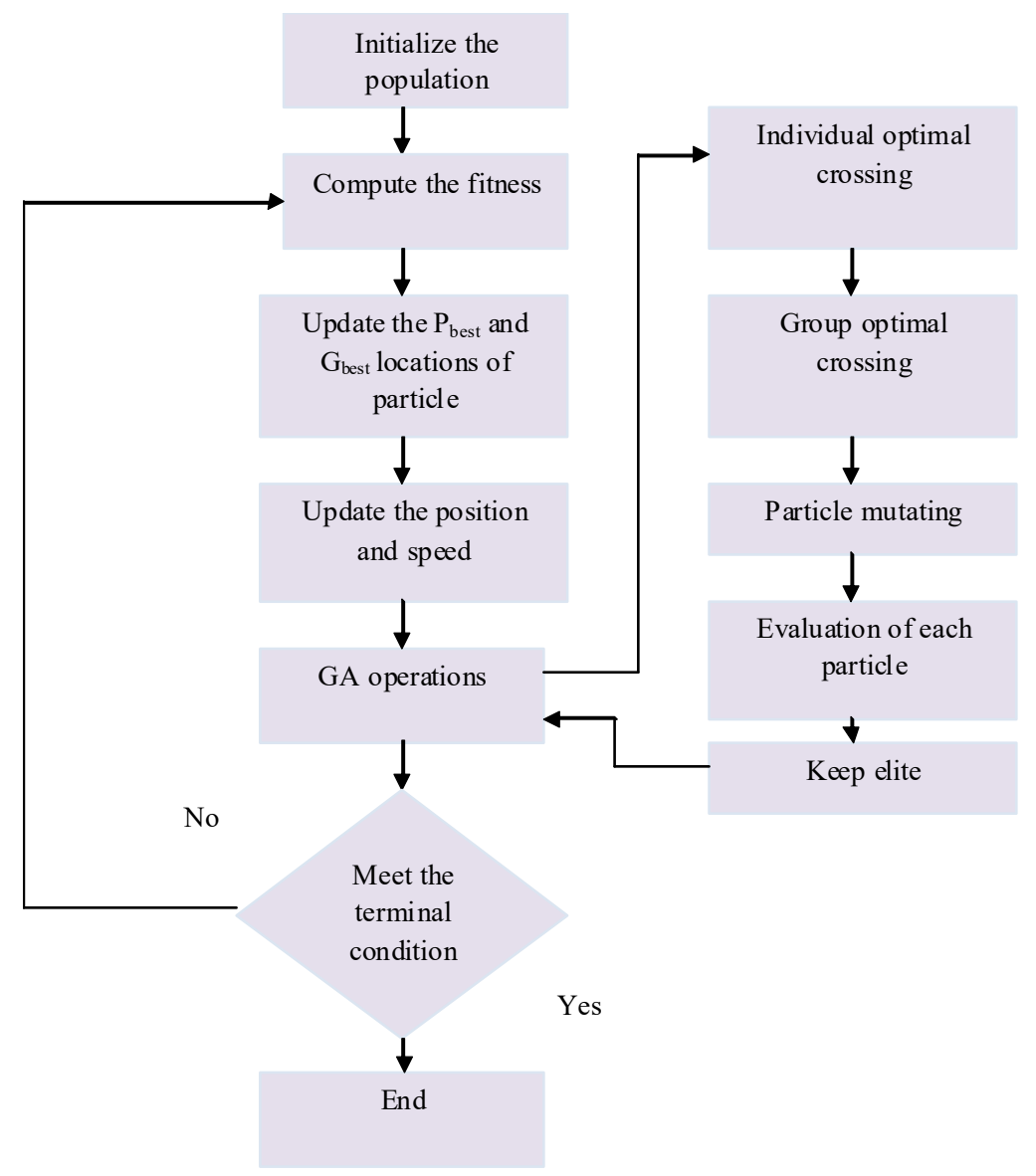

Fig. 1. Process model of proposed Hybrid PSO-GA technique

Table 1: Performance analysis of proposed and conventional algorithms in IEEE 30 bus system

\begin{tabular}{llll}
\hline Methods & GA & PSO & proposed method \\
\hline Fuel cost (\$/h) & 601.24 & 799.41 & 596.96 \\
VD & 1.06 & 0.91 & 1.64 \\
Ploss $_{\text {Lmax }}$ & 9.26 & 6.776 & 6.602 \\
Emission (ton/h) & 0.136 & 0.149 & 0.126 \\
\hline
\end{tabular}

Table 2: Performance analysis of proposed and conventional algorithms in IEEE 57 bus system

\begin{tabular}{llll}
\hline Methods & GA & PSO & proposed method \\
\hline Fuel cost (\$/h) & 828.49 & 830.2223 & 830.288 \\
VD & 4.922 & 4.488 & 4.484 \\
Ploss & 0.484 & 0.298 & 0.298 \\
Lmax $_{\text {Emission (ton/h) }}$ & 0.284 & 0.243 & 0.242 \\
\hline
\end{tabular}

Table 3: Performance analysis of proposed and conventional algorithms in IEEE 118 bus system

\begin{tabular}{llll}
\hline Methods & GA & PSO & proposed method \\
\hline Fuel cost (\$/h) & 826.8471 & 828.29 & 827.78 \\
VD & 4.622 & 4.2768 & 4.262 \\
Ploss & 0.44 & 0.2978 & 0.266 \\
L $_{\max }$ & 0.261 & 0.2401 & 0.246 \\
Emission (ton/h) & 0.24 & 0.124 & 0.124 \\
\hline
\end{tabular}




\section{Conclusion}

In this work, a novel integration of the GA algorithm and PSO was developed. The developed method was implemented effectively on IEEE 30, 57, and 118-bus test systems during the minimization of single and multi-objective functions. The most important characteristic of the developed method is its capability to produce numerous solutions about searching additional cavernous about candidate solutions, that might comprise global optimum. The outcomes attained by this paper shows that the developed algorithm was superior to the conventional techniques at acceptable levels of environmental, technical, and economic advantages. These important technical advantages possess annual minimization of power losses which was attained using the proposed method for the IEEE 30, 57, and 118-bus test systems. The developed method tends to minimum emission levels in environmental concerns. Moreover, the developed method encompasses a robust solution over variation in population size and the utmost iterations evaluated with conventional methods.

\section{Compliance with Ethical Standards}

Conflicts of interest: Authors declared that they have no conflict of interest.

Human participants: The conducted research follows the ethical standards and the authors ensured that they have not conducted any studies with human participants or animals.

\section{References}

[1] Zhao Yuan, Mario Paolone,"Properties of convex optimal power flow model based on power loss relaxation", Electric Power Systems Research, Volume 186, September 2020.

[2] Shuijia Li, Wenyin Gong, Ling Wang, Xuesong Yan, Chengyu Hu,"Optimal power flow withimproved adaptive differential evolution", Energy, Volume 1981 May 2020.

[3] Hossein Saberi, Turaj Amraee, Cuo Zhang, Zhao Yang Dong," A heuristic benders-decomposition-based algorithm for transient stability constrained optimal power flow", Electric Power Systems Research, Volume 185, Aug.2020.

[4] Fariba Zohrizadeh, Cedric Josz, Ming Jin, Ramtin Madani, Somayeh Sojoudi," A survey on conic relaxations of optimal power flow problem European Journal of Operational ResearchIn press, Available online 27 January 2020.

[5] Warid Warid,"Optimal power flow using the AMTPG-Jaya algorithm", Applied Soft Computing, Volume 91June 2020.

[6] Mengxia Wang, Ming Yang, Xueshan Han,"Optimal power flow considering transient thermal behavior of overhead transmission lines", International Journal of Electrical Power \& Energy Systems, Volume 114January 2020.

[7] MATPOWER Available: http://www.pserc.cornell.edu//matpower.

[8] Shaheen AM, Farrag SM, El-Sehiemy RA. MOPF solution methodology. IET Generation, Transmission \& Distribution.

[9] J. H. Holland, "Adaptation in natural and artificial systems," Ann Arbor vol.6, pp.126-137,1975.

[10] J. Huang, W. Li, and M. Ding, "Block-type high temperature gas cooled reactor reloading pattern optimization using genetic algorithm," Qiangjiguang Yu Lizishu/high Power Laser \& Particle Beams 29,2017.

[11] J. Kennedy and R. Eberhart, "Particle swarm optimization[C]," Proc of the IEEE Int Conf on Neural Networks. Piscataway: IEEE Service Center 12, 1941-1948 (1995).

[12] Garg and Harish, "A hybrid PSO-GA algorithm for constrained optimization problems," Applied Mathematics \& Computation, vol. 274, pp.292- 305.

[13] Chithra.S and R.Madahana Kumari,"Economic Emission Dispatch in Renewable Energy Systems using FireFly Algorithm", Journal of Computational Mechanics, Power System and Control (JCMPS), Volume 1, Issue 1, October 2018.

[14] SK.Mahammad Shareef and Dr.R.Srinivasa Rao,"A Hybrid Learning Algorithm for Optimal Reactive Power Dispatch under Unbalanced Conditions", Journal of Computational Mechanics, Power System and Control (JCMPS), Volume 1, Issue 1, October 2018

[15] T.C.Subramanyam,S S Tulasi Ram and J B V Subrahmanyam,"HGAGWO: A Multi-Objective Optimal Positioning and Sizing of Fuel Cells in DG Systems", Journal of Computational Mechanics, Power System and Control (JCMPS), Volume 1, Issue 1, October 2018 